\title{
Research on the Corresponding Relationship between the Key Points of Body and Clothing Structure Design
}

\author{
Shumin $\mathrm{Li}^{1}$ \\ ${ }^{1}$ Jiangxi Institute of Fashion Technology, Nanchang, Jiangxi, 330201
}

KEYWORDS: Human Keys Points, Clothing Structural Design, Corresponding Relationship

\begin{abstract}
This paper mainly detailed analysis of the key parts of the body, while the basis for determining the position of the key points in the costume design to clothing design and structure lines for analysis and research. Costume design based on human need to draw a structure to design and tailoring, in the design of the human body needs to be patterned as a basis for science and rationality.
\end{abstract}

\section{Introduction}

In Garment Design, a board generally planar structure design, one is directly on the human body model for draping. This crop is often directly on the mannequin,, more intuitive way to feel the beauty of the clothes, but the real cost required to operate relatively large. Our current widely used flat structure design, mainly because the method is simple and convenient, in addition, will not result in higher costs. However, this method lacks human involvement, making the design of the clothing and the body is missing out fit. Every design clothing is inseparable from the human body, especially the type of tight-fitting clothes. Clothing is designed to better show the beauty of the human body, so you want to realize the value of clothing, you need to pay attention to points, lines and clothing, in addition, will also take into account the human factor, especially in the key body points, hard to design each piece of clothing.

\section{Review of the Key Parts of the Body from Measurement Points}

Fashion design in addition to considering the beauty of the clothing itself should also be considered outside of the body structure, which has spread from where the ancestors of view. Human thorax, the size of the pelvis, as well as with the neck shoulder, arm their relationship chest cannot be ignored, in general, is that we do not see the waist, but it plays an important role at the time of human movement, waist It would normally be moderately enlarged, so that the wearer does not cause shortness of breath problems. Further, when the twist at the waist will make the pelvis and chest are in motion, making it the center of gravity constantly change with, whereby you show the human dynamic beauty.

Length: distance between points below the neck until a predetermined place; (2) Back Length: the distance between the point and the cervical spine at the waist; (3) the predecessor length: shoulders to the distance between the waist; (4) Sleeve: the distance between the shoulder and wrist endpoints; (5) long pants: from the side of the body measured from the waist to the ankles between; (6) waist length: the distance between the waist and hips; (7) shares in the long-: waist and the distance between the hip bump; (8) Chest: the milk point as a base, soft tape measure level around the circle of data; (9) waist: the waist the finest point as the measurement range, and the elbow 
waist level measurement point as a starting point, use a soft tape measure level around the circle obtained data; (10) hip: the greater trochanter point as a starting point, at the level of the hip most protruding position around the circle of data; (11) the hip: waist and hip for one half of the level around the circle; (12) neck circumference: soft tape measure along the necks, side neck point, neck-point level around the circle; (13) full shoulder width: the distance from one side of the shoulder along the neck between the points until the other side; (14) chest width: starting from the right side of the chest through the armpit to the other side armpit width; (15) back wide: starting from the right axillary back through the distance of the end of the left armpit, (Figure 1).

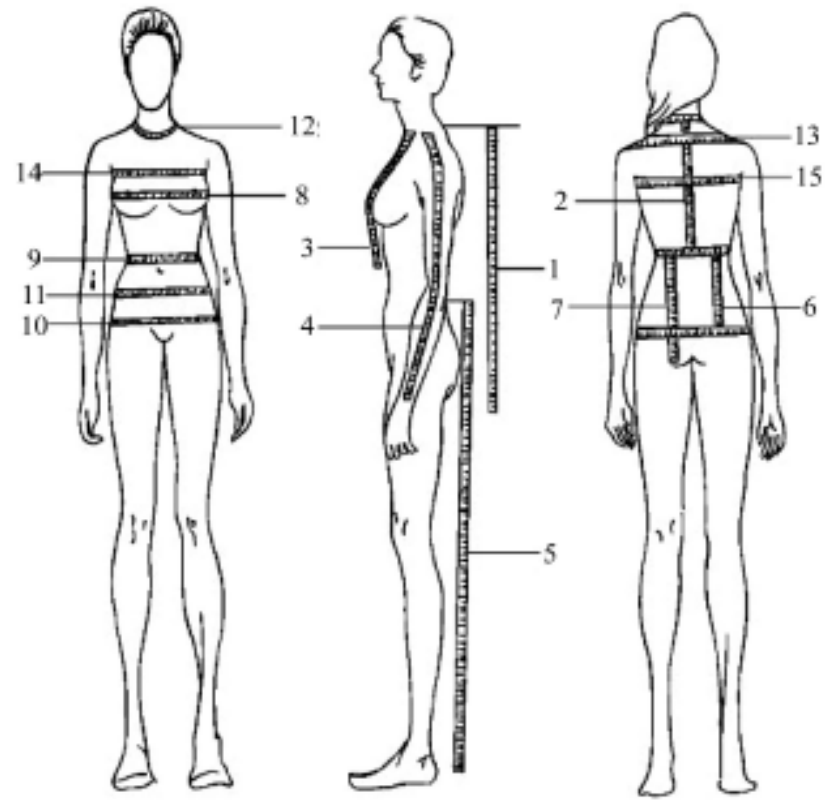

Fig.1 Measurement Points

\section{The Relationship between the Body and Clothing of the Key Points}

Neck collar and costume design: neckline design focus is on the bottom of the human neck, above the collarbone, usually in terms of the wider top narrow bottom. We watched the neckline can find the structure of the human neck was low after the high non-straight state on one side, and the tectonic cause the body back neck - chest - shoulder angles are generating change. Therefore, in the design of the collar, when taking into account the moderate expansion of the collar to prevent neck restricted Also it would be appropriate to put a high collar, collar or the system set up stations lapel. Some people's neck is short, so designers need to do is lower the neckline, so that the wearer will be able to give a longer neck illusion. Some head and neck slightly forward, we should pay attention to the clothing designer collar is slightly moved forward, so that makes the length of the collar becomes longer, the other to the rear panel of the collar positions increased. One shoulder perfect structure for the overall structure of clothing is indivisible, shoulder with shoulder stitching three sides connected to form a structure having a curved arch, suitable clothing enhances the effect of the human body, in this structure in the area of clothing called "wrinkle shoulder" typically adult male tilt angle of $20^{\circ}$, the inclination angle of the shoulder adult woman mean $22^{\circ}$. Mankind forward's body structure has led to a decline in the peak chest, the other leading to the center of the waist and the front armpit point is not in the same straight line, and the front shoulder seam Norwegian clothing, but also to make appropriate adjustments to their respective front and rear, namely before long and short. 
For ordinary physique population, the most critical location of their clothing is at the center of the shoulder. Under normal circumstances, it is not obvious before bending shoulder, shoulder bend more obvious, so the garment sewing process should pay attention to the rear portion of the garment extra income into account, avoid human scapula be bound to improve comfort clothing. Human shoulder, a total of three categories, namely, flat shoulder, slipped shoulder, left shoulder missing. The first category of people with high shoulder lapel clothing would be more appropriate, because it can produce a certain angle collar flat shoulder, so that the wearer's shoulder more three-dimensional sense; for the second shoulder crowd size, the designers have to try to cover the shoulder line, is usually a wing collar, or by looking at the shoulder pads to make more high-rise, avoid selecting station collar; shoulder As for the third category of people, their clothing has three kinds of approach: 1 large lapels, raglan sleeve design 2, 3 at the side of the shoulder pads...

The whole structure of the human body, the upper body is the most flexible part of the highest frequency, and clothing in the sleeve portions, respectively. Elbow bent forward but not bent backwards special structure makes it has a slight curvature of the upper limb in a natural state. Sleeve type design relies on upper limb morphology. Usually by the sleeve $60^{\circ}, 90^{\circ}$, the former includes a sleeve or two sleeves, which only two sleeves. Armhole corresponds to the body's armpits (moderately relaxed position), the main sleeve cage can be divided into three categories: First, the appropriate body and sleeves, and second, the body armpit clothes at deepening three armhole depth is unchanged by the height of the sleeve cap sleeve fertilizer to make changes to determine the tightness of the garment.

Human chest located below the collarbone. Costume design are usually very valued here and through here modified to increase the overall aesthetic of clothing. But some clothing more neutral, that is at the waist and hips are not obvious sexuality, do not charge more the province of the clothing, the show is relaxed sporty.

The ups and downs of the state of the body back to play an important influence in the fashion design process. The key components of the costumes are the ups and downs on the back of the shoulder blades and center. In order to meet the body back configuration, designers usually increases in the garment at shoulder province, and some poor clothing fabrics, to increase comfort back seams to ensure the wearer's back level, but also enhance the sense of the human back curves. Some people exists humpback problems, designers need to use more than the fat suit cover this defect.

Belly and waist in the middle part of the clothing for the performance of the structure is very important, including the person's abdomen below the rib cage, above the pubic bone all boneless parts. Half of the more gentle on the abdomen, the lower half position due to the presence of subcutaneous fat to make them more prominent obviously, surrounded by semicircular trench. Clothing structural efforts need to focus on the waist, the waist is more stout for some people, if not at the waist of the dress to do some adjustments, thick waist naturally lead to poor results, and will also make the body and abdomen waist fat is tightened, the aesthetic shape is a failure, the other people waist thick coat to avoid stuffed under clothing to prevent the body out of type.

Lower Extremity receives the full weight of the body, compared to the more robust and upper limbs, lower limbs corresponds clothing pants. Human thigh are more concerned than other parts stout, transverse therefore crotch pants should be located according to the body to relax properly, of course, cannot be too wide to avoid loose, nor too tight, it will be tied to the wearer, to take into account comfort degree and beauty. The knee is one of the sites that people use higher frequencies, so the knee pants should be appropriate to relax, avoid obstruction knee, while also preventing the reduction of pants beauty. On people of different leg shape is concerned, whether it is short-legged 
or thick legs, are able to use clothing to avoid weaknesses in the choice of clothing should pay attention to this point, the leg does not look good for the people, you must not wear short skirts or shorts, but it should be increased waist pants or skirt, legs to look for the overall image of the wearer extra points.

\section{Conclusion}

Clothing anthropogenic structure but beyond human, designers must first understand the human body and clear structure of the various state, only better fulfill costume design. Garment pattern design process, the designer is important to have a good knowledge of the structure of the human body and the clothing as an important tool to identify clothing fabric points, lines, and complete the structural work cut out the garment. Weaknesses, fully embodies the human body.

\section{Reference:}

[1] Zhou Yue. Correspondence between the Key Body and Clothing Structure Design [J]. Modern Silk Science and Technology, 2010,04: 6-9.

[2] Zhou Sumeng. Technology and Properties of Zhou Sumeng. PTT New Fibers [D]. Donghua University, 2011.

[3] Qi Xingxiang. Fit Evaluation Model based on Personalized Virtual Mannequin Clothing [D]. Donghua University, 2011.

[4] Liu Huan. Reconstruction Based on Stereo Vision More Flexible Airbag Human Abdomen [D]. Donghua University, 2014.

[5] Su Junqiang. Individual Young Women Model based on Local Characteristics of Generation Rule Research [D]. Suzhou University, 2014.

[6] Xia Ming. Based on Analysis of the Female Body and Elliptic Fourier Personalized Custom Prototype Research [D]. Donghua University, 2015.

[7] Zhao Jijun. Effect of Three-Dimensional Virtual Technology based on A Single Stand Catchy Line Arc Length Factors [D]. Collar. Donghua University, 2013.

[8] Hu Haiming. Based on A Combination of Relations with Cuff Sleeves Women Modeling [D]. Zhejiang University of Technology, 2013. 